\title{
THE EFFECTS OF CONCURRENT VISUAL FEEDBACK ON THE CONTROL OF SWIMMING INTENSITIES ABOVE THE ONSET OF BLOOD LACTATE ACCUMULATION
}

original paper

(1) University School of Physical Education in Wroclaw

DOI: https://doi.org/10.5114/hm.2018.77324

\section{STEFAN SZCZEPAN ${ }^{1}$, KRYSTYNA ZATON ${ }^{1}$, JACEK BORKOWSKI ${ }^{2}$}

${ }^{1}$ Department of Swimming, University School of Physical Education in Wrocław, Wroclaw, Poland

${ }^{2}$ Department of Physiology and Biochemistry, University School of Physical Education in Wrocław, Wroclaw, Poland

\section{ABSTRACT}

Purpose. Effective control of swimming speed can increase training effectiveness and therefore enhance training adaptations. The aim of the present study was to investigate the effects of concurrent visual feedback on swimming speed at an exercise intensity above the onset of blood lactate accumulation (OBLA).

Methods. The study involved 12 healthy male and female swim team members. The participants executed two $200-\mathrm{m}$ freestyle trials within an individually predetermined swim time matching an exercise intensity above OBLA (exercise zone 4). In one trial, no additional information was provided, whereas concurrent visual feedback was given on swimming speed in the other.

Results. Concurrent visual feedback narrowed the discrepancy between the predetermined and obtained swimming times $(p<0.001)$. This improvement in swimming time (accuracy) showed a negative correlation with the increase in post-trial lactate concentration. The correlation between the difference in the predetermined and obtained times and the increase in lactate was $R=-0.46$ when no visual feedback was provided but $R=-0.64$ with visual feedback, with the latter result statistically significant $(p=0.024)$.

Conclusions. Concurrent visual feedback allows swimmers to consistently maintain a predetermined swimming speed and therefore keep the specified exercise intensity as evidenced by the increase in lactate concentration. This can result in more effective execution of training goals and thereby enhance training outcomes.

Key words: visual feedback, concurrent feedback, pacing in swimming, swimming speed control, high intensity OBLA

\section{Introduction}

The goal of training is to enhance physiological function and improve skills needed to achieve success. This is achieved by executing specific tasks of increasing intensity. Training in each intensity/zone is used to improve performance and/or swimming technique at higher intensities. These differences in exercise intensity are commonly categorized into 5 training zones [1]. The first and second zones are characterized as aerobic exercise, which in the swimming context involves swimming at speeds beneath the anaerobic threshold. Swimming at this intensity stimulates muscle repair and regeneration and improves aerobic capacity. Aerobic capacity is of critical importance as it allows athletes to perform a larger volume of exercise repetitions at greater work intensity (e.g. during interval training) [2]. The third training zone (aerobic/anaerobic) is performed at an intensity between the anaerobic threshold and the athlete's maximum oxygen uptake $\left(\mathrm{VO}_{2} \mathrm{max}\right)$. Training at this intensity can improve both the aerobic and anaerobic metabolic systems. The fourth zone involves swimming at $\mathrm{VO}_{2} \max$, whereas the fifth zone is above the $\mathrm{VO}_{2}$ max threshold and is treated as anaerobic exercise. The last 2 zones are sustained by the anaerobic system and produce significant concentrations of blood lactate, in which the threshold is termed as the onset of blood lactate accumulation (OBLA). It is worth noting that training above the OBLA initiates muscle tissue changes, leading to an improvement in aerobic metabolism, as lactate accumulation is associated with an increase in the concentration of adenosine monophosphate

Correspondence address: Stefan Szczepan, Department of Swimming, University School of Physical Education, al. Paderewskiego 35, 51-612 Wroclaw, Poland, e-mail: stefan.szczepan@awf.wroc.pl

Received: May 19, 2017

Accepted for publication: January 10, 2018

Citation: Szczepan S, Zaton K, Borkowski J. The effects of concurrent visual feedback on the control of swimming intensities above the onset of blood lactate accumulation. Hum Mov. 2018;19(4):49-55; doi: https://oi.org/10.5114/hm.2018.77324. 
(AMP) in myocytes. The increased concentration of AMP activates complex changes in the AMP-dependent kinase enzymes, including an increase in the oxidative resynthesis pathway of adenosine triphosphate (ATP) [3]. Hence, exercise performed at an intensity above the OBLA is of significant importance as it can considerably improve the fitness level. Incorrectly determined swimming intensity, for instance intensity above the OBLA, can result in under- or overtraining. Recurrent training performed at an incorrect intensity can also create inadequate movement habits [4]. This can lead to poor development of aerobic capacity, negatively affecting the efficiency of anaerobic processes and performance of high-intensity exercise [5].

Swimming speed is a primary indicator of exercise intensity [6]. It is possible to improve physiological adaptation to training by controlling the swimming speed. For example, swimming at speeds in the training zones below or above the anaerobic threshold can be used for lactate tolerance training [7]. In order to verify the absolute intensity of swimming, a coach can measure the time and distance to calculate the swimming speed. However, a more difficult task is to determine the relative intensity of swimming, which is a measure that depends more on the swimmer's current fitness level. An important role of a coach is to provide athletes with information on the duration and/or proper swimming speed or intensity of an exercise task $[8,9]$. This is typically performed via verbal communication. However, environmental conditions may distort the exchange of information. In swimming, the use of swim caps is widespread and the athlete's head is frequently submerged beneath the water. Various methods have been introduced to provide feedback, from communicating via wireless headphones [10] to the use of a timer submerged at the bottom of the pool [11, 12]. Nevertheless, none of these methods can convey real-time information on the proper swimming speed. Therefore, developing a method that can improve swimming speed control can optimize training and therefore performance, particularly among young or inexperienced swimmers who are unable to maintain the correct swimming speed or intensity. One of such methods is providing external feedback about the duration and/or swimming speed or intensity of an exercise.

The swimmer responds to information such as feedback from exteroreceptors and proprioreceptors. During swimming, external feedback is transmitted in 3 forms: words, images, and practical actions. Concurrent visual feedback was deemed by many researchers and trainers as an important part of training, including a method that allows for effective acquisition and improvement of skill or error prevention [13]. The feedback was divided into 2 types: intrinsic (integral) and extrinsic. Intrinsic feedback is the sensory information arising as a result of physical activity by means of sensory mechanisms (exteroreceptors and proprioreceptors). Extrinsic feedback (augmented feedback) is formed after the completion of a motor activity and is transmitted by a third party [14]. The functions of extrinsic feedback consist in filling in information gaps, motivating, and reinforcing [15]. There are several types of feedback, categorized in accordance with the time of its transmission: concurrent feedback (provided during a motor task, concerning continuous information), immediate feedback (provided during a motor task, concerning discrete information), and delayed feedback (transmitted after the completion of a motor action) [14]. Owing to the existence of factors disrupting feedback in a swimming environment (noise, head submerged in the water, swimming caps, etc.), visual feedback provided in real time could prove to be a very powerful method. One such device is the Lider optical fibre (Kuca Ltd., Poland), giving augmented feedback to the swimmer. The aim of the present study was to investigate the effects of concurrent visual feedback on swimming speed during exercise performed at an intensity above OBLA.

\section{Material and methods}

\section{Participants}

The study involved 12 healthy male and female swim team members (age, $17.7 \pm 3.4$ years; body height, $180.8 \pm 9.6 \mathrm{~cm}$; body mass, $68.8 \pm 10.8 \mathrm{~kg}$; training experience, $7.4 \pm 1.7$ years; personal best in $200-\mathrm{m}$ freestyle, $136.44 \pm 14.7 \mathrm{~s}$ ). All participants were informed about the nature and circumstances of the study.

\section{Procedure}

The experiment proper consisted of two 200-m freestyle swimming trials performed in a standard $25-\mathrm{m}$ swimming pool at a speed corresponding to training zone 4 , as this zone primarily involves anaerobic exercise [1]. An individual swim time for each participant was determined by using personal bests in the 200-m freestyle from the current season. The Pansold Test für Schwimmer (Mesics GmbH, Germany) software was used to determine whether the relative exercise intensity was similar among the participants. The Pansold software automatically calculates the per- 

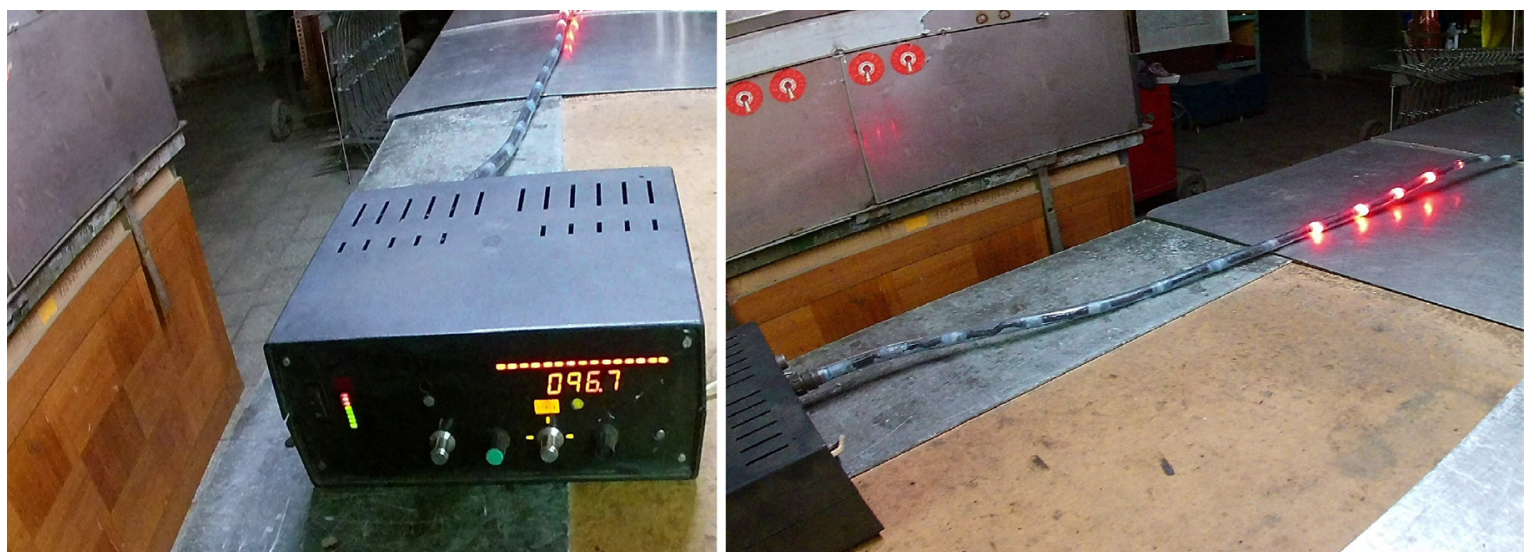

Figure 1. The Lider device (controller with arterial tube with light-emitting diodes) before installation at the bottom of the swimming pool

sonal best, and, as a result, defines times of swimming for 5 zones: (1) recovery zone, (2) oxygen zone, (3) aerobic/anaerobic zone, (4) $\mathrm{VO}_{2}$ max zone, (5) anaerobic zone.

Each trial was preceded by a warm-up, in which the 200-m freestyle was swum at a speed corresponding to intensity zone 1 (low intensity exercise below the anaerobic threshold) [1]. A cool-down followed in order for the heart rate to decrease to resting values. In both trials, a water start was employed. Each participant was to swim the $200-\mathrm{m}$ distance as closely as possible to their predetermined time. In the first trial, the subjects swam without any information provided on the swimming speed. In the second trial, real-time visual feedback was provided with the use of the device outlined below. The trials were separated by a $24-\mathrm{h}$ interval and performed at the same time of day. The swimmers were asked to maintain a normal diet, refrain from alcohol and caffeine, and avoid any strenuous physical activity during the study period [16].

\section{Swimming speed control}

Visual feedback was provided with the use of a Lider device (Kuca Ltd., Poland) (Figure 1) mounted at the bottom of the pool. The device includes transparent tubes that house sequential light-emitting diodes. In this way, a beam of light is scrolled across the bottom of the pool at the speed calculated for each test participant. All of the subjects had previously trained with the Lider at least once.

\section{Measurements}

Blood lactate concentration was measured 3 times: before the warm-up (baseline), 3 min after the warm-up (pre-trial), and 3 min after completing the trial (posttrial), when the lactate concentration had a maximum value [17]. Lactate assays were treated as a measure of the relative intensity of the applied exercise. They were not repeated because post-exercise restitution was not investigated. The concentration of lactate was determined via an enzymatic method (Hydrex, Italy) in which $20 \mathrm{ml}$ of blood was collected from the participant's fingertip and immediately diluted in a cold isotonic solution containing $\mathrm{NaF}$ and $\mathrm{NaCl}$. Heart rate was also recorded immediately before and after each trial with a heart rate monitor (Polar Electro, Finland). The swim time was measured electronically with the Colorado Time System (Colorado Time Systems, USA) with an accuracy of $0.01 \mathrm{~s}$.

\section{Statistical analyses}

Data were processed with the Statistica 9.0 software package (StatSoft, USA). In the first analysis, Student's $t$-test was used to determine the statistically significant differences between the analysed variables (predetermined and obtained swim time, pre- and posttrial lactate concentration, and pre- and post-trial heart rate) in both trials (with and without visual feedback). Using the $t$-test is a proper way to establish differences between 2 groups [16]. Pearson's correlation coefficients were then calculated, with the changes in lactate and heart rate analysed in relation to the difference in the predetermined and obtained swim times. The results were presented as means and standard deviations.

\section{Ethical approval}

The research related to human use has been complied with all the relevant national regulations and institutional policies, has followed the tenets of the Declaration of Helsinki, and has been approved by the authors' institutional ethics committee. 


\section{Informed consent}

Informed consent has been obtained from all individuals included in this study.

\section{Results}

The results for time, swimming speed, lactate concentration, and heart rate for both conditions (with and without feedback) are presented in Table 1. The differences between the predetermined and obtained swim times $(\Delta t)$ were smaller when concurrent visual feedback was provided. When expressed as absolute terms (the measure of inaccuracy), the obtained time in relation to the predetermined time significantly $(p<0.001)$ decreased from $\bar{x}=3.33 \pm 1.92 \mathrm{~s}$ to $\bar{x}=$ $0.25 \pm 0.62 \mathrm{~s}$. For lactate concentration and heart rate, no statistically significant changes were observed.

The observed improvement in swimming time accuracy (as the reduction in the difference between the pre-

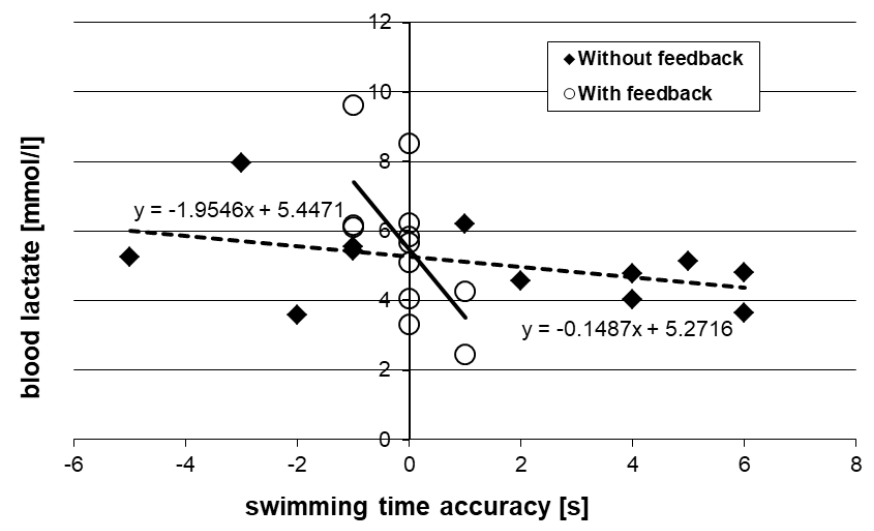

Figure 2. The relationship between swimming time accuracy and the increase in lactate concentration (with and without visual feedback) determined and obtained swimming times) showed a negative correlation with the increase in lactate concentration. The correlation between swimming accuracy (time difference) and the increase in lactate was $r=-0.46$ for the trial without visual feedback and $r=$ -0.64 in the test with visual feedback (Figure 2), albeit only the correlation in the visual feedback condition was statistically significant $(p=0.024)$.

\section{Discussion}

The main goal of a swim coach and swimmer during a training cycle is to attain a specific outcome within a specific time frame. Appropriate workloads, particularly in the form of training intensity, are an important factor in not only enhancing physiological function but also mastering a specific technique at a given swimming speed. One rational method to determine training intensity is by adopting an empirical classification system, such as the use of training zones, as in the present study [1]. Among various solutions, the covered time needed to transgress a given distance or heart rate, and post-training blood lactate concentrations offer a relatively simple way to evaluate the absolute and relative intensity [5]. For this reason, these temporal and physiological measures have been adopted in the real world setting [18]. Nonetheless, these methods do possess a common fault in that such information regarding intensity is frequently delivered only at the end of exercise. In the swimming context, the lack of concurrent feedback therefore reduces training effectiveness by not allowing necessary adjustments to the swimming speed. While real-time heart rate monitoring is now possible via wireless communication, it is worth not-

Table 1. Pre- and post-trial measures with and without visual feedback

\begin{tabular}{|c|c|c|c|c|c|}
\hline \multirow{2}{*}{ Variables } & \multicolumn{2}{|c|}{ No feedback } & \multicolumn{2}{|c|}{ Feedback } & \multirow[t]{2}{*}{$p$} \\
\hline & $\bar{x}$ & $S D$ & $\bar{x}$ & $S D$ & \\
\hline Predetermined time (s) & 144.25 & 11.77 & 144.25 & 11.77 & \\
\hline Obtained time (s) & 147.15 & 10.51 & 145.00 & 11.26 & \\
\hline$\Delta \mathrm{t}(\mathrm{s})$ relative value & 1.33 & 3.43 & -0.08 & 0.62 & 0.21 \\
\hline$\Delta \mathrm{t}(\mathrm{s})$ absolute value & 3.33 & 1.92 & 0.25 & 0.62 & $0.00007^{*}$ \\
\hline Speed $(\mathrm{m} / \mathrm{s})$ & 1.37 & 0.11 & 1.39 & 0.12 & 0.09 \\
\hline Pre-La (mmol/l) & 2.69 & 1.30 & 2.69 & 1.30 & \\
\hline Post-La (mmol/l) & 7.80 & 1.84 & 7.47 & 2.14 & \\
\hline$\Delta \mathrm{La}(\mathrm{mmol} / \mathrm{l})$ & 5.10 & 1.11 & 5.61 & 2.03 & 0.19 \\
\hline Pre-HR (bpm) & 84.50 & 17.80 & 74.30 & 27.30 & \\
\hline Post-HR (bpm) & 167.00 & 22.00 & 171.00 & 24.10 & \\
\hline$\Delta \mathrm{HR}(\mathrm{bpm})$ & 82.50 & 20.30 & 90.50 & 28.50 & 0.33 \\
\hline
\end{tabular}

La - lactate concentration, HR - heart rate

* statistically significant difference $(p<0.01)$ 
ing that the aquatic environment often impairs proper operation. Furthermore, heart rate is determined not only by exercise intensity but also by other factors, such as emotional state, which may confound results. Again, while lactate concentrations provide a more objective assessment of training intensity, such information is available only upon exercise completion.

As analysed in the present study, one method that can effectively deliver information on exercise intensity in a swimming context is visual feedback. This form of feedback is considered very efficient particularly in difficult environmental conditions [19]. As a way of controlling mechanical performance, visual feedback can deliver real-time continuous information during movement execution [14]. Besides controlling intensity, this form of feedback is also useful in the prevention and correction of movement errors [15]. The primary objective of this study was to investigate the effects of concurrent visual feedback on swimming speed during exercise performed at an intensity above OBLA.

Interestingly, the trial involving the concurrent visual feedback condition showed a significant improvement in swimming accuracy $(p<0.001)$, suggesting not only maintenance of proper exercise intensity but also more precise movement execution. It is probable, too, that some of the participants were not able to perform at the specified intensity until visual feedback was introduced. These results correspond with those obtained by Gonzalez et al. [11], who presented a new electronic system for the control of swimming speed. The authors presented a prototype system for the control of swimmers, especially when precise speed is required for specific measurement tests. The electronic system was based on a series of high luminosity lightemitting diodes, spaced $1 \mathrm{~m}$ apart, put inside a plastic pipe placed at the bottom of the pool. In this study, visual feedback was provided by a Lider device (Kuca Ltd., Poland) mounted at the bottom of the pool. However, sequential light-emitting diodes were placed every $1 \mathrm{~cm}$ along the pool ( $25 \mathrm{~m})$, as opposed to every $1 \mathrm{~m}$. As a result, the swimmer received continuous visual information. Also, Pérez et al. [12] maintained that a swimmer's pace control was affected by the type of feedback provided, the swim speed elected, and the size of the swimming pool. Swim time depends on feedback regarding such factors as chronometer, coach, or no feedback. However, significant differences were observed only between the group of coach-chronometer feedback and no feedback condition $(p<0.01)$, while the interaction between the swimmer and feedback condition was not significant $(p>0.05)$. In both citied studies, the swimmers received information about time/speed that supported their performance.

An additional issue of this study was the swimmer's physiological response to the exercise in intensity above the OBLA. This difference in intensity was confirmed by a greater difference in the pre- and posttrial lactate concentration in the visual feedback condition (5.61 $\mathrm{mmol} / \mathrm{l})$ than when no visual feedback was provided $(5.10 \mathrm{mmol} / \mathrm{l})$. Nevertheless, there was no statistically significant difference $(p=0.19)$, though with tendency to significance while the number of subjects increased. Furthermore, improved swimming accuracy showed a negative correlation with the increase in post-trial lactate concentration. The correlation coefficient between the difference in the predetermined and obtained times and the increase in lactate was $R=-0.46$ when no visual feed back was provided but $R=-0.64$ with visual feedback, with the latter result statistically significant $(p=0.024)$. With regard to heart monitoring, mean $\Delta \mathrm{HR}$ equalled $82.50 \mathrm{bpm}$ in the group without feedback and $90.50 \mathrm{bpm}$ in the group with feedback. The difference was not statistically significant ( $p=0.33$ ). Similarly, no statistically significant differences $(p=0.09)$ were found in attained speed: $1.37 \mathrm{~m} / \mathrm{s}$ for the group without feedback, $1.39 \mathrm{~m} / \mathrm{s}$ for the group with feedback.

These results show that concurrent visual feedback allows swimmers to consistently maintain a proper time of the task (significantly smaller differences between the predetermined and obtained swim times, $\Delta \mathrm{t}$ absolute value $p=0.00007$ ). As a result, exercise intensity turned out higher, which was estimated by lactate concentration (greater difference in lactate concentration, $\Delta \mathrm{La} p=0.19$, though not statistically significant). Despite no statistically significant difference in lactate concentration, time accuracy was negatively correlated with the increase of lactate concentration $(R=-0.64$, statistically significant, $p=0.024)$. That means that a subject swam with more accuracy with an increase of lactate concentration which was statistically significant $(p=0.024)$. This enabled the performance of the exercise at a proper intensity above OBLA, which was the aim of the task. The main findings of the study were to provide a proof of the concept that real-time information (concurrent visual feedback) on proper duration/swimming can help effective execution of training goals (performance of exercise with proper intensity).

The obtained results regarding the beneficial effects of swimming pace control on lactate concentration are convergent with a study by Turner et al. [20], who used an audio system to control the swimming speed. They applied an audio-pacing device that was pro- 
grammed to provide a sound signal not only at the end of the 200-m split but also at the end of each 25-m pool length, thus accurately regulating swimming velocity. The audio-paced system increased achievement among young competitive swimmers, especially when lactate was accumulated in excess.

Fatigue causes deterioration in the movement quality control [21]. In the presented study, lactate concentrations of $7 \mathrm{mmol} / \mathrm{l}$ and above suggested that the adopted swimming intensity was maintained in zone 4 (above the OBLA) and most likely above the anaerobic threshold.

An application value of the present study is that visual feedback helps maintain proper speed, which provides a suitable training stimulus by preserving exercise intensity and improving performance. Maintaining exercise intensity was evaluated by lactate concentration. The method can allow swimmers not only to meet training goals but also to augment training on the basis of their own individual needs [22]. It is worth to note that although Lider is a technical device, it can be used in swim training without restricting the swimmers' movements, which could potentially affect their achievement. Additionally, it is possible to run many beams of light on one lane. This allows several athletes to swim in one lane during one task. They can swim one after another with the same time setting. This results in one lane being used by swimmers of a similar ability level. Often, this is not a problem because the coach can appropriately divide the group. Nevertheless, the device providing concurrent visual feedback can be used at different levels of swimmers' age to improve their performance and support training aims achievement.

The results of the project should be interpreted with caution owing to its limitations. Split times during the 200-m trials were not presented. In order to evaluate the control of swimming intensity on a $200-\mathrm{m}$ bout, it would be essential to provide data about intermediate times for each 50 or $100 \mathrm{~m}$. Future studies should thus include split time data. However, each subject was to swim the $200-\mathrm{m}$ distance as closely as possible to their predetermined time, and after each trial all participants reached a lactate concentration of more than $7 \mathrm{mmol} / \mathrm{l}$, which indicated effort above OBLA. As for the limitations of the visual information system, the device does not control changes in swimming velocity due to the push of the wall during the start and turns, which clearly modifies swimming velocity control.

\section{Conclusions}

Concurrent visual feedback allows swimmers to consistently maintain a predetermined swimming speed (swimming accuracy) and therefore preserve the specified exercise intensity as evidenced by the increase in lactate concentrations. This can result in a more effective execution of training goals and thereby enhance training outcomes.

\section{Acknowledgments}

The authors express their sincere thanks to the constructor of the Lider device (Kuca Ltd., Poland), Mr. Witold Szajnert, for providing tools for the experiment.

\section{Disclosure statement}

No author has any financial interest or received any financial benefit from this research.

\section{Conflict of interest}

The authors state no conflict of interest.

\section{References}

1. Bompa TO, Haff GG. Periodization. Theory and methodology of training, $5^{\text {th }}$ edition. Champaign: Human Kinetics; 2009.

2. Koral J, Oranchuk DJ, Herrera R, Millet GY. Six sessions of sprint interval training improves running performance in trained athletes. J Strength Cond Res. 2018; 32(3):617-623; doi: 10.1519/JSC.0000000000002286.

3. Jessen N, Sundelin EI, Møller AB. AMP kinase in exercise adaptation of skeletal muscle. Drug Discov Today. 2014;19(7):999-1002; doi: 10.1016/j.drudis.2014.03.009.

4. Siniscalchi MJ, Cropper EC, Jing J, Weiss KR. Repetition priming of motor activity mediated by a central pattern generator: the importance of extrinsic vs. intrinsic program initiators. J Neurophysiol. 2016;116(4): 1821-1830; doi: 10.1152/jn.00365.2016.

5. Kenney WL, Wilmore JH, Costill D. Physiology of sport and exercise, $5^{\text {th }}$ edition. Champaign: Human Kinetics; 2012.

6. Scruton A, Baker J, Roberts J, Basevitch I, Merzbach V, Gordon D. Pacing accuracy during an incremental step test in adolescent swimmers. Open Access J Sports Med. 2015;6:249-257; doi: 10.2147/OAJSM.S84906.

7. Sperlich B, Zinner C, Heilemann I, Kjendlie PL, Holmberg HC, Mester J. High-intensity interval training improves $\mathrm{VO}_{2}$ peak, maximal lactate accumulation, time trial and competition performance in 9-11-year-old swimmers. Eur J Appl Physiol. 2010;110(5):10291036; doi: 10.1007/s00421-010-1586-4.

8. Micklewright D, Angus C, Suddaby J, St Clair Gibson A, Sandercock G, Chinnasamy C. Pacing strategy in schoolchildren differs with age and cognitive development. Med Sci Sports Exerc. 2012;44(2):362-369; doi: 10.1249/MSS.0b013e31822cc9ec.

9. Chinnasamy C, St Clair Gibson A, Micklewright D. Effect of spatial and temporal cues on athletic pacing in schoolchildren. Med SciSports Exerc. 2013;45(2):395402; doi: 10.1249/MSS.0b013e318271edfb. 
10. Zatoń K, Szczepan S. The impact of immediate verbal feedback on the improvement of swimming technique. J Hum Kinet. 2014;41:143-154; doi: 10.2478/hukin2014-0042.

11. Gonzalez V, Sanchis E, Villalobos M, Brizuela G, Llana S, Tella V. A new electronic system for the control of swimming speed. In: Chatard JC (ed.), Biomechanics and medicine in swimming IX. Saint-Étienne: Publications de l'Université de Saint-Étienne; 2002; 67-69.

12. Pérez P, Llana S, Brizuela G, Encarnación A. Effects of three feedback conditions on aerobic swim speeds. J Sports Sci Med. 2009;8(1):30-36.

13. Andrieux M, Proteau L. Observational learning: tell beginners what they are about to watch and they will learn better. Front Psychol. 2016;7:51; doi: 10.3389/ fpsyg.2016.00051.

14. Schmidt RA, Lee TD. Motor learning and performance. From principles to application, $5^{\text {th }}$ edition. Champaign: Human Kinetics; 2013.

15. Anderson DI, Magill RA, Sekiya H, Ryan G. Support for an explanation of the guidance effect in motor skill learning. J Mot Behav. 2005;37(3):231-238; doi: 10.3200/ JMBR.37.3.231-238.

16. Thomas JR, Nelson JK, Silverman SJ. Research methods in physical activity, $7^{\text {th }}$ edition. Champaign: Human Kinetics; 2015.

17. Goodwin ML, Harris JE, Hernández A, Gladden LB. Blood lactate measurements and analysis during exercise: a guide for clinicians. J Diabetes Sci Technol. 2007; 1(4):558-569; doi: 10.1177/193229680700100414.

18. Morouço PG, Marinho DA, Keskinen KL, Badillo JJ, Marques MC. Tethered swimming can be used to evaluate force contribution for short-distance swimming performance. J Strength Cond Res. 2014;28(11):30933099; doi: 10.1519/JSC.0000000000000509.

19. Szczepan S, Zatoń K, Klarowicz A. The effect of concurrent visual feedback on controlling swimming speed. Pol J Sport Tourism. 2016;23(1):3-6; doi: 10.1515/pjst2016-0001.

20. Turner AP, Smith T, Coleman SG. Use of an audio-paced incremental swimming test in young national-level swimmers. Int J Sports Physiol Perform. 2008;3(1):6879; doi: 10.1123/ijspp.3.1.68.

21. Rodríguez DS, del Valle Soto M. A study of intensity, fatigue and precision in two specific interval trainings in young tennis players: high-intensity interval training versus intermittent interval training. BMJ Open Sport Exerc Med. 2017;3(1):e000250; doi: 10.1136/ bmjsem-2017-000250.

22. Szczepan S, Zaton K. Validation of the new visual swimming pace control system in real-time. Cent Eur J Sport Sci Med. 2017;19(3):93-104; doi: 10.18276/ cej.2017.3-09. 